\title{
Emerging Trends of Outsourcing Business to Cloud Computing Services: A Perspective Study
}

\author{
Amjad Hudaib ${ }^{1}$, Mohammad Alnabhan' ${ }^{2}$ Osama Harfoushi ${ }^{3}$, \\ Ruba Obiedat ${ }^{3}$, Omar Adwan ${ }^{1}$, Waed Adham ${ }^{1}$ \\ ${ }^{1}$ Department of Computer Information Systems, King Abdullah II School for Information Technology, \\ The University of Jordan, Amman, Jordan \\ ${ }^{2}$ Department of Computer Science, Jerash University, Jerash, Jordan \\ ${ }^{3}$ Department of Business Information Technology, King Abdullah II School for Information Technology, \\ The University of Jordan, Amman, Jordan \\ Email: Ahudaib@ju.edu.jo, m.alnabhan@jpu.edu.jo, o.harfoushi@ju.edu.jo, \\ r.obiedat@ju.edu.jo, adwanoy@ju.edu.jo, waed-adham@live.com
}

Received November 8, 2013; revised December 8, 2013; accepted December 15, 2013

Copyright (C) 2014 Amjad Hudaib et al. This is an open access article distributed under the Creative Commons Attribution License, which permits unrestricted use, distribution, and reproduction in any medium, provided the original work is properly cited. In accordance of the Creative Commons Attribution License all Copyrights (C) 2014 are reserved for SCIRP and the owner of the intellectual property Amjad Hudaib et al. All Copyright (C) 2014 are guarded by law and by SCIRP as a guardian.

\begin{abstract}
Developments in service oriented architecture (SOA) have taken us near to the once fictional dream of forming and running an online business, such commercial activity in which most or all of its commercial roles are outsourced to online services. The novel concept of cloud computing gives a understanding of SOA in which Information Technology assets are provided as services that are extra flexible, inexpensive and striking to commercial activities. In this paper, we concisely study developments in concept of cloud computing, and debate the advantages of using cloud services for commercial activities and trade-offs that they have to consider. Further we presented a layered architecture for online business, and then we presented a conceptual architecture for complete online business working atmosphere. Moreover, we discuss the prospects and research experiments that are ahead of us in realizing the technical components of this conceptual architecture. We conclude by giving the outlook and impact of cloud services on both large and small businesses.
\end{abstract}

\section{KEYWORDS}

Cloud Business; Cloud Services; Cloud Computing; Traditional Software Developing; Software Engineering Life Cycle; Service Oriented Architecture

\section{Introduction}

The knowledge of forming and administrating a business on the Internet is not novel. Large manufacturers, financial institutions and banks are amongst the leading in using the electronic network abilities to perform business-to-business (B2B) connections by using technologies such as Electronic data Interchange EDI was first established in 1993. By the time with the starter of the websites and the swift growth of web consumers in the early 1990s, businesses for instance eBay and Amazon were amongst the primary players to the business-toconsumer (B2C) model of electronic commerce. As the Internet is a speedy, convenient-to-use and inexpensive medium which fascinates millions of consumers online at any time, currently there are very rare companies that do not have presence on Internet, and there are numerous small and medium businesses (SMBs) for instance retail shops that exclusively propose their products and services completely online.

When we look at the empowering technologies, B2C and $\mathrm{B} 2 \mathrm{~B}$ electronic commerce have promoted from countless inventions in the Web and Internet. When moving to dynamic update of page content and the introduction of XML from the static content delivery to form the major development in the track to new interoperable and competent administration of electronic businesses. A key feature of employing technologies of the Web 1.0 age is 
that nearly all the backend IT systems are formed, functioned and sustained by the business holders. Inspired by commercial quickness, working competence, price decrease and better affordability, through the past decade, companies have taken benefit of Business process outsourcing (business processing outsourcing) [1,2]. In this type of outsourcing, companies depute selected of the company's non-core commercial operations for instance IT processes to third-party outdoor units that majors in those roles. It is assessed that by 2011 the global market for Business Process Outsourcing will reach $\$ 677$ billion [3]. Cloud computing is a model for allowing ubiquitous, suitable, on-demand network access to a shared pool of configurable computing resources (e.g., networks, servers, storage, applications, and services) that can be quickly provisioned and unrestricted with negligible management energy or service provider communication [4].

Till lately, subcontracted amenities were not essentially achieved online. Business processing outsourcing has turn out to be attractive for both small and large businesses with the beginning of facility focused on computing [5] and precisely Web 2.0 technologies and Web services. This has allowed proposing of business process roles as online services and energetically fetching clienteles via the Internet [6]. It is projected that business processing outsourcing characterizes about $25 \%$ of the overall services market [3].

The subsequent evolutionary tendency in this space is cloud computing. Cloud computing states to the proposing of software and hardware properties as services crossways (distributed) IT assets [7]. As a comparatively novel idea, cloud computing and associated technologies have quickly increased drive in the IT world. In this paper, we study how developments in cloud computing influence the procedures of forming and administrating companies on the Internet. In specific, we examine the inquiry of whether the technology is equipped to permit business holders to form and run a business by means of services over the Web. We state to this as an "online business" in which maximum or all of its roles are subcontracted to online services. It should be compared to the idea of "online enterprise" [8] which frequently mentions to making a provisional coalition or grouping of businesses to discourse firm needs with an importance on incorporating technologies, dissemination of capabilities and responsibilities and knowledge distribution. Infrastructure services provided by cloud service provider's permits number of users to have access towards a large number of compute facilities extremely easily [9].

The rest of this paper gives a short survey of developments in cloud computing, and by an example situation, we have highlighted the trade-offs that companies have to ponder in moving to cloud services. Further, we debate the necessities of an atmosphere for forming and admini- strating online businesses, and present business architecture for such an environment. We further present encounters that are forward of us in presenting such a situation and offer a viewpoint of the influence of cloud services on businesses.

\section{Cloud Computing: A Short Survey}

Cloud computing has appeared as the accepted development and combination of improvements in numerous grounds together with grid computing, service oriented architecture distributed computing, web services, and utility computing [7]. The worth and innovation of cloud computing originates from contributing assets in an inexpensive, accessible and flexible way that is reasonable and striking to IT clienteles and technology financers. We present an outline to study and review developments in cloud computing. It contains of four dimensions: cloud service customers, cloud services, multi-tenancy as an enabler and public vs private clouds. This cloud computing is a novel technology and emerged as super paradigm for hosting and offering services over the internet. It is attractive to businesses and it diminishes the requirement for users, and permits a company to start from the small and increase resources [10].

\subsection{Traditional Software Development through the Cloud}

Traditional software development through the cloud by renting the cloud and start developing using online editors (see Figure 1) by going through the following traditional software engineering life cycle:

1) Software specification and analysis: a requirements specification for a software system, is a complete description of the behavior of a system to be developed and may include a set of use cases that describe interactions the users will have with the software. In addition it also contains non-functional requirements. Non-functional requirements impose constraints on the design or implementation (such as performance engineering requirements, quality standards, or design constraints).

The software requirements specification document enlists all necessary requirements that are required for the project development. To derive the requirements we need to have clear and thorough understanding of the products to be developed. This is prepared after detailed communications with the project team and customer.

2) Software design: is the process by which an agent creates a specification of a software artifact, intended to accomplish goals, using a set of primitive components and subject to constraints. Software design may refer to either "all the activities involved in conceptualizing, framing, implementing, commissioning, and ultimately modifying complex systems" or "the activity following 


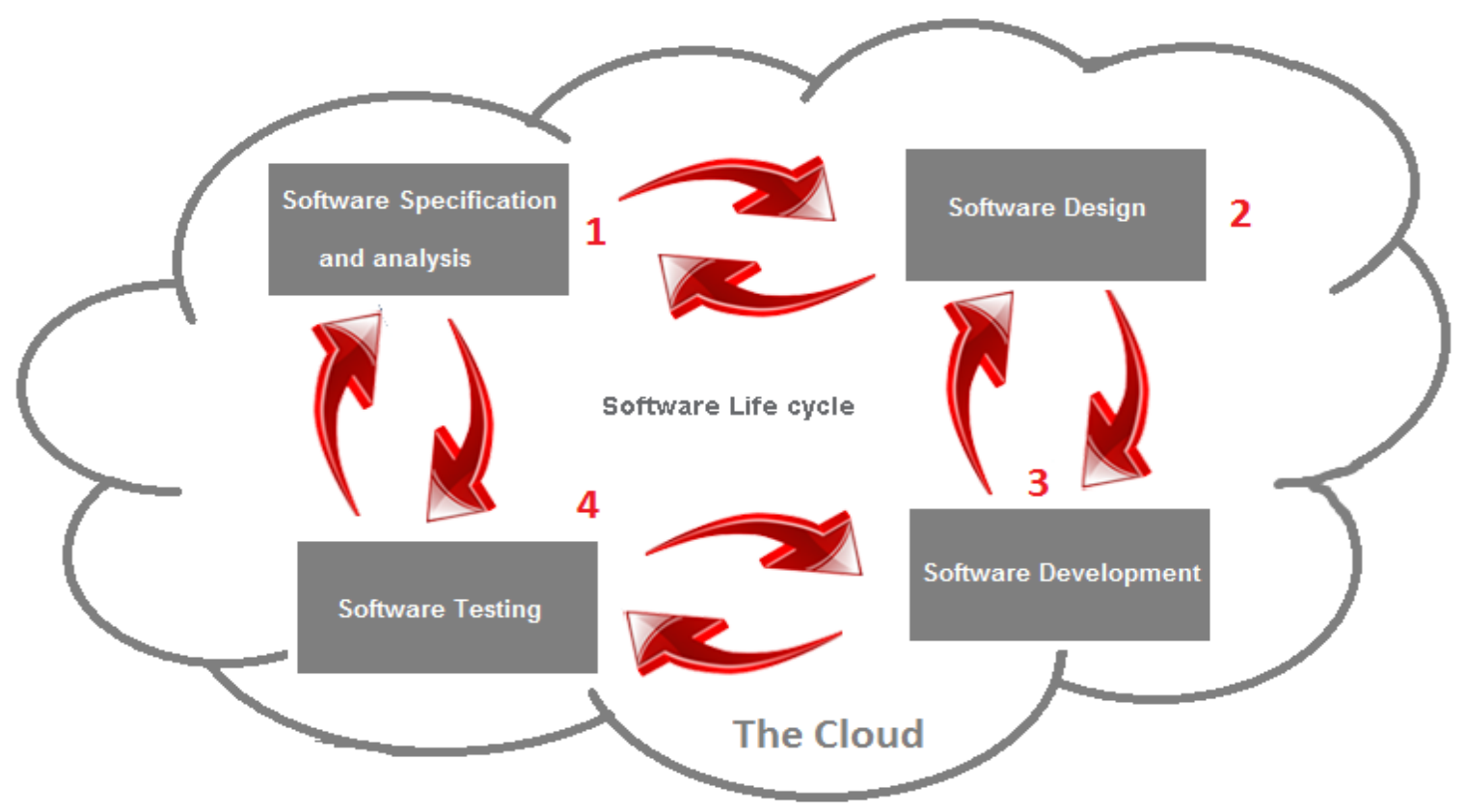

Figure 1. Software development through the cloud.

requirements specification and before programming, as... [in] a stylized software engineering process.”

Software design usually involves problem solving and planning a software solution. This includes both lowlevel component and algorithm design and high-level, architecture design.

3) Software development: (also known as application development, software design, designing software, software application development, enterprise application development, or platform development) is the development of a software product. The term "software development" may be used to refer to the activity of computer programming, which is the process of writing and maintaining the source code, but in a broader sense of the term it includes all that is involved between the conception of the desired software through to the final manifestation of the software, ideally in a planned and structured process. Therefore, software development may include research, new development, prototyping, modification, reuse, reengineering, maintenance, or any other activities that result in software products.

4) Software testing: is an investigation conducted to provide stakeholders with information about the quality of the product or service under test, Software testing can also provide an objective, independent view of the software to allow the business to appreciate and understand the risks of software implementation. Test techniques include, but are not limited to the process of executing a program or application with the intent of finding software bugs (errors or other defects).

Software testing can be stated as the process of validating and verifying that a computer program/applica- tion/product:

- meets the requirements that guided its design and development,

- works as expected,

- can be implemented with the same characteristics,

- satisfies the needs of stakeholders.

Software testing, depending on the testing method employed, can be implemented at any time in the software development process. Traditionally most of the test effort occurs after the requirements have been defined and the coding process has been completed, but in the Agile approaches most of the test effort is on-going. As such, the methodology of the test is governed by the chosen software development methodology.

\subsection{Cloud New Services}

As supported by the idea of "everything as a service” [11] numerous products are currently presented as services beneath the umbrella of cloud computing. These services are classified into four categories:

1) Infrastructure as a service (IaaS): hardware resources (such as storage) and computing power (CPU and memory) are presented as services to clients. This allows companies to charge these resources instead of spending money to purchase dedicated networking equipment and servers. Every so usually businesses are payable for their usage subsequent a convenience computing model, where usage of assets is measured. As instances in this category, EC2 for computing power, SQS for network communication and Amazon1 offers S3 for storage for small companies and different consumers. HP 
FCS2 (Flexible Computing Services) delivers storage infrastructure and computing as services for businesses [12].

2) Database as a service (DaaS): a new particular sort of storage is provided database ability as a service. Samples of service providers are Google, Force.com Amazon SimpleDB, Microsoft SSDS4database and platform. DaaS on the cloud frequently accepts a multi-tenant architecture, where the data of various consumers is reserved in the identical physical table. Similarly, in best cases, the database structure is not interactive.

3) Software as a service (SaaS): software applications are provided as services on the Internet relatively than as software packages to be bought by different clienteles. One of the original suppliers in this category is Salesforce.com provided its CRM application as a service. Other cases include Microsoft online CRM and SharePoint, Adobe Photoshop and Adobe Premiere on the Web or Google web-based office applications. Commercial applications in this category might require a monthly subscription per user (salesforce.com) or can be payable per use. There is no certified software issue cycle, and the purchaser is unrestricted from applying updates as this is controlled by the service provider. Buyer data is saved in the cloud, actually based on DaaS.

4) Platform as a service (PaaS): PaaS provides services to maintain the complete application development lifecycle together with design, debugging, facilities on the Internet operation and support of rich Web applications, deployment and implementation and testing. Mostly Internet browsers are used as the development setting. Cases of platforms in this category are Google App Engine7Microsoft Azure Services platform6, Bungee Connect platform9, salesforce.com and Internet Application Development platform8. PaaS permits SaaS consumers to develop standalone Web-based applications and also develop add-ons, reuse other services and develop collaboratively in a team. In this category, there are other kinds of abilities that are delivered as services that are delivered in the cloud such as Provided management, monitoring. In monitoring as a facility, a third-party provider (e.g., Red Hat Command Center) witnesses SaaS applications or the IT network of an enterprise on behalf of a customer with respect to SLAs and reports performance metrics to the buyer. One more significant service that is delivered on the cloud is people as services. Offering of services by people, e.g., their encoding skills per hour on the net, is maybe as old as the Web itself. On the other hand, what is different in the cloud is there are people specializing in SaaS or PaaS platforms and providing consultation for companies that requirement to use or modify SaaS results or mix solutions from numerous SaaS providers.

\subsection{Public vs Private Clouds}

It can be argued that the cloud is the outcome of normal conversion of the IT structure of companies over the past decade. The old IT structure was grounded on having devoted assets for every business unit in a company. This structure leads to waste of IT resources and under-utilization due to resource disintegration and uneven circulation of workload. To overwhelm this, companies have applied adaptive infrastructure techniques [13]. These comprise using online solutions to discourse the under-utilization problem completed with automation techniques to decrease the important labor costs of IT processes. This kind of cloud is called a "private" cloud as it is privately owned by companies. Cases of this kind are clouds preserved by producers such as Boeing or GM.

Alternatively, there are other cloud providers (e.g., those provided by Salesforce.com Google, Amazon, and Microsoft) for community use. Several of these clouds e.g., those provided by Google and Amazon, are certainly additions of their private clouds that are provided to the public. There are also cloud providers such as Salesforce.com that have formed and provided cloud services only for public use. It is exciting to notice that companies and large businesses are mostly the owners and consumers of private clouds, while public clouds are used by smaller companies and millions of individual consumers.

Further to cloud sellers, who own and control cloud services, there are other providers called out-clouders (re-sellers). Out-clouders are becoming popular and trying to obtain and re-sell idle computing assets of companies with private clouds [14].

\subsection{Cloud Service Consumers}

Addition to the coarse-grained categorization of cloud consumers as companies, small to medium businesses and individual consumers, it is valuable to recognize and study several types of consumers of cloud services. Understanding the target consumers of cloud services and their necessities permits identifying what sort of services can be used by which consumers. Generally, cloud clients are of the following types: Managers and business owners IT administrators, individual (business) consumers and finally software developers. Table 1 illustrates the supply of several cloud consumers for many cloud services.

\subsection{Multi-Tenancy as an Enabler}

Multi-tenancy talks about allocating resources among consumers and applications. It is ideal over single-tenancy in cloud services due to advance utilization primary to cost reduction. Businesses usually have thousands of customers but normally work a diversity of soft- 
Table 1. Cloud consumer's vs cloud services.

\begin{tabular}{|c|c|c|c|c|c|}
\hline Consumer type & IaaS & DaaS & SaaS & PaaS & $\begin{array}{l}\text { Others: such as (monitoring } \\
\text { as a Service) }\end{array}$ \\
\hline IT administrators & $\begin{array}{l}\text { Use to deploy images } \\
\text { of existing software }\end{array}$ & $\begin{array}{l}\text { Configure to } \\
\text { store data }\end{array}$ & Only for administration use & Not available & $\begin{array}{c}\text { Monitoring as a service } \\
\text { (to setup and monitor SLAs) }\end{array}$ \\
\hline Software developers & $\begin{array}{l}\text { May use to } \\
\text { deploy software }\end{array}$ & Store data & $\begin{array}{l}\text { Mainly to browse and find existing } \\
\text { services to reuse and add-ons }\end{array}$ & $\begin{array}{l}\text { Main consumers } \\
\text { of PaaS }\end{array}$ & Integration as a service \\
\hline $\begin{array}{l}\text { Managers and } \\
\text { business owners }\end{array}$ & Not available & Not available & $\begin{array}{l}\text { Occasional consumers to manage } \\
\text { their business }\end{array}$ & Not available & $\begin{array}{l}\text { Monitoring as a } \\
\text { service(dashboards), may } \\
\text { employ people as services }\end{array}$ \\
\hline Business consumers & Not available & Not available & $\begin{array}{l}\text { Main consumers of SaaS, may } \\
\text { perform simple configuration tasks } \\
\text { and use add-ons }\end{array}$ & Not available & Not available \\
\hline
\end{tabular}

ware environments and applications. Therefore, in private clouds multi-tenancy is usually about having multiple applications and environments deployed on shared resources. If public cloud providers offer PaaS, then a variation of application environments are also supported. In this case, multi-tenancy methods need to allow sharing resources among volumes of applications and customers.

\section{Cloud Retail as an Online Business}

Let's take an example of a small imaginary company named Cloud Retail, from the group of SMBs with a few hundred employees through the country. This company basic product is to designs and sells stylish and environmental friendly clothing and accessories. They use bond manufacturers but sell straight to their customers via their Website and catalog. Their fundamental ability is environmental friendly product design rapidly exploiting on tendencies in the marketplace. Cloud Retail use software in-house for certain purposes, for instance customer relationship management (CRM), human resources, and their customer-facing web site. They have an IT department which keeps the IT substructure inside the company. This IT infrastructure has developed more difficult and costly to maintain as it has developed with the company. It now comprises specialized storage and network equipment, dozens of servers, and an ever-growing list of software, much of it to guarantee smooth and secure process of the company and the website.

Evolving Cloud Retail into an online business using cloud services is the ideal scenario for them. Cloud Retail can take benefit of several current cloud services together with HR, CRM, IT infrastructure and the hosting and operation of their website. Following are some benefits for using Cloud Services: 1) Companies can avoid huge start up investments in software and hardware resources, 2) Companies can reduce ongoing operational, upgrade and maintenance costs, 3) By using Cloud Services companies can scale up and down network capacity, hardware and cost based on demand, 4) Moreover businesses can high availability compared to in-house solu- tions for individual-consumer maintained resources and small businesses, and 5) Finally companies can also be beneficial from access to a diversity of features provided as SaaS and software applications that otherwise Cloud Retail would have to purchase separately.

On the other hand, the possible hazards of using cloud services comprise: 1) companies can bear loss of direct control of resources and software, e.g., website structure and operations staff for Cloud Retail, 2) better liability risk due to security breaks and data leaks accordingly using shared outside resources, 3) reduced dependability since the service providers might go out of business, data retrieval issues and disturbing business continuity.

\section{Online Business Operating Environment}

A large and increasing number of services are accessible most of which target individual consumers and small companies (the long tail of service customers). The extensive variation and low cost of cloud services delivers an extraordinary opportunity and financial motivation for companies to move their IT infrastructure to services in the cloud. There is a persistent requirement for an environment that permits SMBs and individual consumers to produce and run an operational business using cloud services. We call this an online business operating environment.

\subsection{Requirements of an Online Business Operating Environment}

An online business operating environment delivers amenities that permit business owners to form their business in a general way: define their business, express their necessities, find and involve cloud services that match their requirements, comprise services if needed, and monitor their business processes over subcontracted services. In specific, it should offer the following sub-environments:

Business definition environment: this should let the business proprietors to define the business, its arrangement (e.g., organization chart), aims and policies in some 
form that can be chased down to the service implementation level and accomplished.

IT services marketplace: this states to an environment where the necessities of customers in terms of IT services can be taken, current services could be publicized, and services to browse and discover existing services are delivered. The services marketplace might backing numerous business models of providing services, e.g., bidding for user tasks, pay-per-use or subscription-based payments.

Business services design integration and composition environment: This refers to an environment where designated services from the marketplace can be organized, composed, new services established if needed and organized to IaaS platforms. Interoperation between services for both functional and non-functional features can be tested and integration solutions established.

\subsection{Online Business Operating Environment: Conceptual Architecture}

Business designs have been widely studied during the last thirty years. Frameworks such as Zachman11 [1] and industry values such as TOGAF13 [15] define enterprise design. In certain, the Zachman framework classifies a number of orthogonal (horizontal and vertical) features. The horizontal layers comprise related (goals and strategies of business), theoretical (high-level design), rational (system-level design) and physical (technology model) meanings for an enterprise. The vertical dimensions classify features that describe each horizontal dimension. Other work also displays how a facility oriented design and application of systems in an enterprise can fit in the Zachman framework [16]. Conversely, these frameworks reflect cases where the IT infrastructure and services are settled and organized as in-house explanations. We present the commercial architecture in a subcontracted service environment as showed in Figure 2 and defined in the following business architecture is described in a subcontracted services setting in four layers: business context, business services delivers for the definition of structure, business processes and IT services, metrics and indicators, business goals, policies and performance strategies. The foremost customers of amenities at this level are business owners and executives who are hardly IT experts. The main functions (supporting or core) of a business such as human resources, payroll, accounting, etc. are distinct as coarse-grained services, stated to as "business services" in the business services layers. Customers such as business/IT experts might define or select the required business services from out-of-box business services blueprints. The IT services layer signifies the services that are delivered in the cloud. To conclude, the business processes layer is the symbol of selection, design, incorporation and arrangement of IT services in the arrangement of workflows that accomplish the necessities of outlined business services. Figure 2 also illustrates the communication of sub-environments of an online business operational environment with the online business architecture, and the clients of numerous layers/sub environments.

\subsection{Realizing the Online Business Operating Environment: Opportunities and Challenges}

To analyze how the existing progresses in SOA, cloud computing and current standards and practices aid in realizing an online business working environment, and recognize the restrictions and tests. Note that in addition the novel and exclusive challenges posed by delivered and using services in the cloud, some of which is reviewed in the following, countless trials of understanding an online business working environment are connected to locating, constituting, assimilating and managing services. Most of
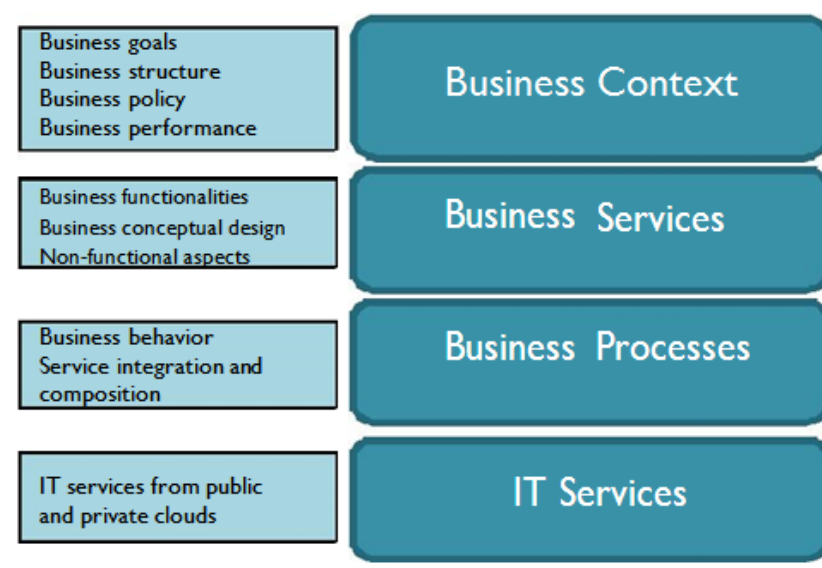

Abstraction Layers

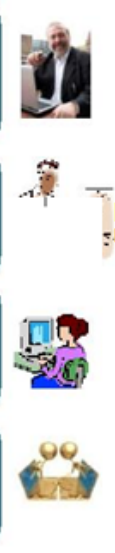

Developers
Deviness users

IT admins

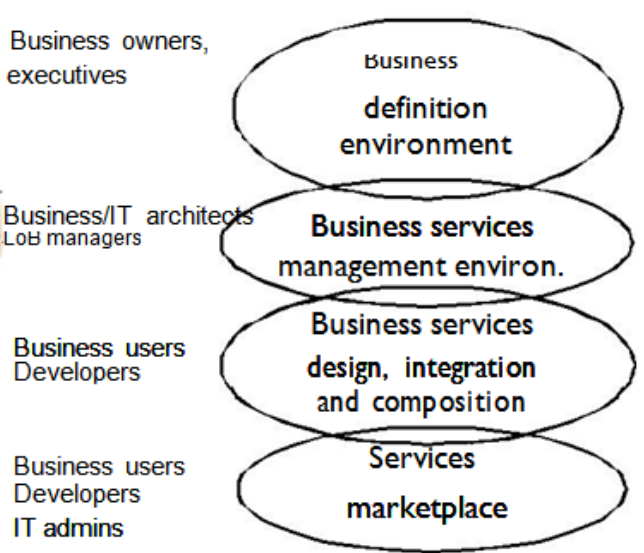

Users Environments

Figure 2. Business architecture in an outsourced services environment. 
these are the same as those known for general services in SOA [5]. In the following, we highlight why current solutions for tackling these difficulties are wanted in the cloud services setting.

Business context layer: the Object Management Group (OMG, www.omg.org) has projected a set of matching business modeling conditions. In specific, the model of business drew in the business motivation modeling (BMM) description (v1.0)13 can be reflected as a standard for the business setting layer. It models a business having elements together with "end" (vision, goals, and objectives of the business), "means" to understand the end (mission, strategy, tactics, and directives with business rules and business procedures) and assessment elements to describe and appraise the enactment of the business. It ought to be noted that for a small to medium businesses not all these workings might be essential, but, these offer rules that can be modified to define a business in an online business scenario.

Business services layer: business services can be separated into three categories: common (found in most businesses such as HR or CRM), industry-specific (found in vertical industries of the same type of business) and company-specific (unique to the given business). The atmosphere has to offer blueprints of business amenities in the above classes, and similarly permit companies to describe business.

Specific business services: these descriptions can be used to discover services from the marketplace that might accomplish the necessities. There is some preliminary work on the description of business services [17], though, a more detailed study is desired to seizure many business-level properties and strategies into this definition.

IT services layer: though there is a big body of work in SOA on IT service description, search and management based on mutually functional and non-functional characteristics [5], the following challenges remain:

Service description and search: a principal challenge is that not all services that are obtainable on the Internet are defined using Web services interfaces (e.g. WSDL) nor are they essentially provided online. Current service search methods are mostly intensive on the interfaces (functional aspects) of services and only support Web services, e.g., UDDI14 and Google [18], or are simply lists with keyword search, e.g., seekda.com.

SLA, data privacy and security concerns: a value of consuming services in the cloud is that the place where customer data is reserved is out of the customer's governor. Presently, there is no provision for instructing exact data safety procedures to service providers.

Business processes and integration layer: even though there are important progresses in service and data integration [19,20] and service configuration [21] and [22] in SOA, difficulties such as how to repeatedly de- termine several Web services (including services with text-based interfaces, people services, etc.) that jointly satisfy a business service. Given that most SaaS customers are business customers (not expert IT people), there is a basic for frivolous resolutions for configuration and integration, e.g., based on advances in mashups [23], to talk these difficulties relatively than only depend on durable resolutions of conformist SOA that are directed for enterprise settings and need skilled consumers' involvement.

\section{Conclusions}

Small companies such as Cloud Retail have already understood the aids of using facilities in the cloud for most non-core operations. Customer's advantage from the costcutting of scale and the extremely improved IT processes of cloud service providers. The chance to evade capital costs and experience expectable expenditures which scale up and down with the existing requirements of the business is very striking. Consumers with irregular or burst practice see incredible assistances, as they merely pay for resources when they are consuming them. Customers with steady procedure arrangements also advantage due to the minor cost of buying services than structuring them internal. Except IT is an essential capability of the business, most clienteles will not be capable to achieve the identical competences inexpensive by doing it themselves. As one illustration, Google's business email solution is, on usual, ten times fewer cheap than internal email solutions.

We foresee that the little price of using cloud computing is an important element of its extensive recognition by different customers, SMBs as well as big businesses. On the other hand, large companies will service a hybrid cloud model in which both public clouds and private clouds are available. Countless firms will run missioncritical applications and store business-sensitive data in the private clouds, while subcontracting their supporting services to the public cloud.

The part that IT staff is playing in the businesses and companies is similarly experiencing a noteworthy revolution due to the thrust for more business processing outsourcing grounded on cloud services. As majority IT staff transfer from commercial IT departments to service providers, the part of remaining staff alters into provision and discussion for service selection, implementation and administration.

In terms of usage of services in the cloud, small SMBs and different consumers will be the leading customers of PaaS, SaaS, IaaS and DaaS. Businesses might demand modification of services as the APIs delivered by service providers might not compromise the flexibility and features they need. Furthermore, they might request exam- 
ples of services to be organized in their private clouds for the sake of reserving data onsite and holding control.

The online business functioning situation for forming and steering online businesses by cloud-based services is an absent part and this research lays the basis of construction for an atmosphere that addresses this persistent requirement for businesses that aim to use cloud services.

\section{REFERENCES}

[1] J. Zachman, "A Framework for Information Systems Architecture,” IBM Systems, Vol. 26, No. 3, 1987, pp. 276-292. http://dx.doi.org/10.1147/sj.263.0276

[2] J. B. Halvey, "Business Process Outsourcing: Process, Strategies, and Contracts,” John Wiley \& Sons, 2007

[3] C. E. Anderson, "Worldwide and US Business Process Outsourcing 2007-2011 Forecast: Market Opportunities by Horizontal Business Process,” 2007.

[4] P. Mell and T. Grance, "The NIST Definition of Cloud Computing (Draft),” NIST Special Publication, Vol. 800, No. 145, 2011, p. 7.

[5] M. P. Papazoglou, "Service-Oriented Computing: State of the Art and Research Challenges," IEEE Computer, Vol. 40, No. 11, 2009, pp. 38-45.

[6] HP, "The Benefits of Combining Business-Process Outsourcing and Service-Oriented Architecture,” 2012.

[7] A. Weiss, "Computing in the clouds," ACM netWorker, Vol. 11, No. 4, 2007, pp. 16-25.

[8] C. Petrie, "Bussler, Service Agents and Virtual Enterprises: A Survey," IEEE Internet Computing, Vol. 7, No. 4, 2003, pp. 68-78. http://dx.doi.org/10.1109/MIC.2003.1215662

[9] J. Ekanayake and G. Fox, "High Performance Parallel Computing with Clouds and Cloud Technologies," Cloud Computing, Springer Berlin Heidelberg, 2010, pp. 20-38.

[10] Q. Zhang, L. Cheng and R. Boutaba, "Cloud Computing: State-of-the-Art and Research Challenges," Journal of Internet Services and Applications, Vol. 1, No. 1, 2010, pp. 7-18. http://dx.doi.org/10.1007/s13174-010-0007-6

[11] S. Robison, "The Next Wave: Everything as a Service, Executive Viewpoint," Retrieved from The next wave: Everything as a service, Executive Viewpoint, 2010. http://www.hp.com/hpinfo/execteam/articles/robison/08ea as.html

[12] N. Carr, "The Big Switch: Rewiring the World," Edison to Google, W. W. Norton Publishing, 2008.

[13] HP, HP Adaptive Infrastructure, 2013.

[14] J. E. Yarmis, "Outclouding: New Ways of Capitalizing on the Economics of Cloud Computing and Outsourcing," AMR Research, Vol. 12, No. 2, 2008, pp. 131-139.

[15] TOGAF, "The Open Group Architecture Framework," Version 8.1.1., 2013. www.togaf.org

[16] M. G. Ibrahim, "Service-Oriented Architecture and Enterprise Architecture,” 2013.

[17] G. E. Scheithauer, "Describing Services for Service Ecosystems," International Workshop on Enabling Service Business Ecosystems, Vol. 2, No. 4, 2008, pp. 23-33.

[18] X. E. Dong, "Similarity Search for Web Services," Proceedings of VLDB, Vol. 4, No. 1, 2004, pp. 372-383.

[19] R. M. Hamid, "Web Services Interoperability Specifications,” IEEE Computer, Vol. 39, No. 5, 2006, pp. 24-32. http://dx.doi.org/10.1109/MC.2006.181

[20] A. E. Halevy, "Data Integration: The Teenage Years," Proceedings of VLDB, Vol. 5, No. 2, 2006, pp. 9-16.

[21] S. A. Dustdar, "A Survey on Web Services Composition," International Journal of Web and Grid Services, Vol. 1, No. 1, 2005, pp. 1-30. http://dx.doi.org/10.1504/IJWGS.2005.007545

[22] A. C. Brogi, "Semantics-Based Composition-Oriented Discovery of Web Services," ACM Transactions on Internet Technology, Vol. 8, No. 4, 2008, pp. 1-39. http://dx.doi.org/10.1145/1391949.1391953

[23] D. S. Benslimane, "Services Mashups: The New Generation of Web Applications," IEEE Internet Computing, Vol. 12, No. 5, 2008, pp. 13-15. http://dx.doi.org/10.1109/MIC.2008.110 